\title{
Radiation Hard Sensors for the BeamCal of the ILC
}

\author{
C. Grah \\ for the FCAL Collaboration \\ Deutsches Elektronen-Synchrotron DESY \\ Zeuthen, D-15738, Platanenallee 6, Germany \\ *E-mail: christian.grah@desy.de
}

\begin{abstract}
BeamCal is an electromagnetic sampling calorimeter in the very forward region of the detectors at the ILC. BeamCal will be hit by a large fraction of electronpositron pairs stemming from beamstrahlung. The sensors used for BeamCal have to withstand very high levels of total ionizing dose. We report on the investigations of radiation hard sensor materials for BeamCal of the FCAL collaboration. Artificial diamond, radiation hard silicon, $\mathrm{SiC}$ and $\mathrm{GaAs}$ sensors are under consideration. Static measurements of the current-voltage characteristics, response to minimum ionizing particles and test beam measurements are part of our investigations.

Keywords: ILC, forward region, BeamCal, CVD diamond, radiation hard silicon, GaAs, CCD, charge collection distance, irradiation
\end{abstract}

\section{Introduction}

The International Linear Collider, ILC, will be a future linear electronpositron accelerator. ${ }^{1}$ Due to the very small beam sizes and high beam intensity at the ILC the creation of beamstrahlung will be a new phenomenon. These beamstrahlung photons are emitted under a very low angle of less than one mrad, but a fraction of the photons can convert into electronpositron pairs which will hit the forward region of the detectors at the ILC. The FCAL Collaboration ${ }^{2}$ develops the systems in the forward region independently from one of the detector concepts. In Fig. 1 a design of the very forward region for the example of the Large Detector Concept, LDC, ${ }^{3}$ is shown for the collider baseline concept of a crossing angle of $14 \mathrm{mrad}$ between the incoming and outgoing beams.

The major systems in this region are LumiCal, which will measure the integrated luminosity to a relative precision of better than $10^{-3}$ using bhabha events, and BeamCal, which will give a fast feedback signal of 


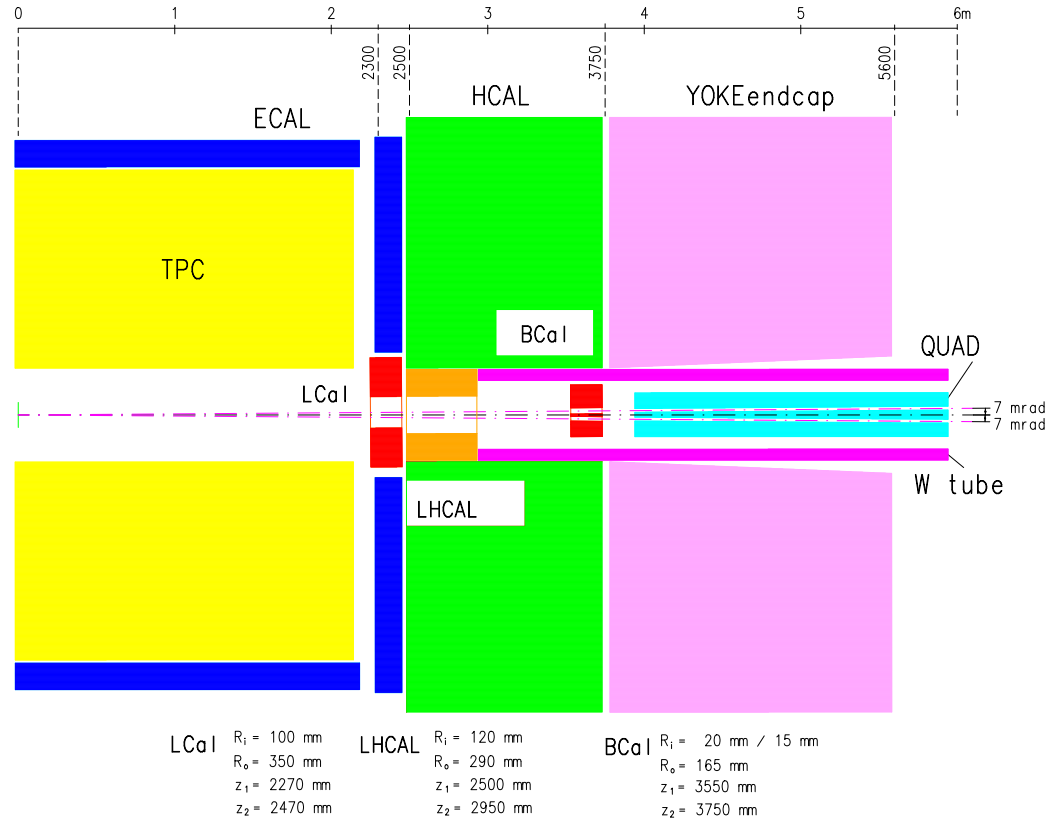

Fig. 1. The forward region of the Large Detector Concept.

the instantaneous luminosity. Both system with also optimize the hermeticity of the detector and guarantee efficient electron detection down to lowest polar angles. Details of the forward region are given in Ref. 4.

\section{The Beam Calorimeter BeamCal}

BeamCal will be a compact electromagnetic calorimeter using tungsten as absorber and a radiation hard sensor material as sensitive layers. Each of the 30 layers of BeamCal will consist of a $3.5 \mathrm{~mm}$ thick absorber layer followed by a sensor layer, which includes a segmented sensor and a readout plane which routes the interconnection lines from the segments to the outer radius of the BeamCal, where the readout electronics will be placed. Fig. 2 shows the expected dose rate per year in the sensor layer with the highes energy deposition, which is after $6 \mathrm{X}_{0}$ in this case. The energy deposition is strongly dependent on the geometry and magnetic field configuration. In the shown example a DID-like magnetic field configuration was chosen, where the magnetic field is parallel to the incoming beam. ${ }^{5}$ According to these GEANT4 simulations the sensors for the BeamCal should be qualified 
for a total ionizing dose of $10 \mathrm{MGy}$ per year. Another requirement for the sensors for the BeamCal is the linearity of the response over at least four orders of magnitude of particle fluences. This arises due to the task of giving efficiently a veto signal of single high energetic particles (e.g. electrons) in the acceptance region of BeamCal on top of the high amount of background from beamstrahlung pairs.

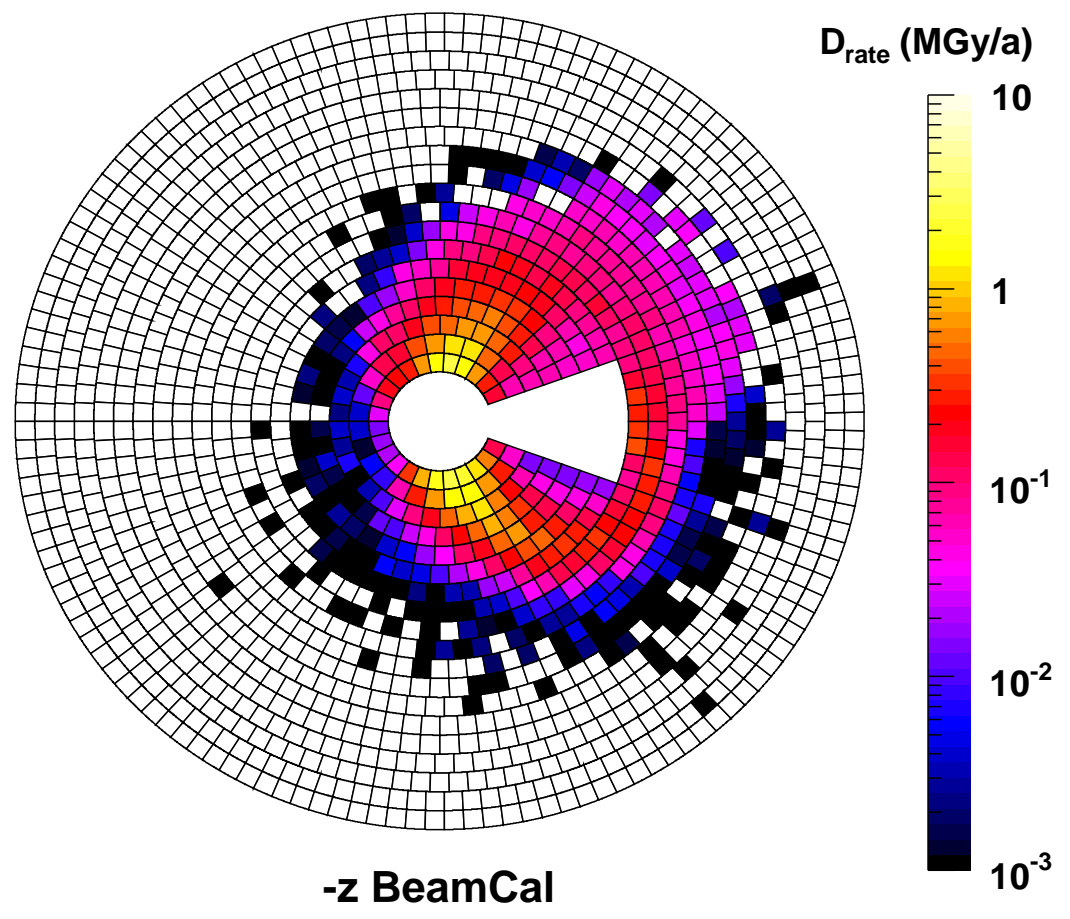

Fig. 2. The expected dose rate for the BeamCal sensor layer with highest energy deposition. The shown case assumes a $100 \%$ running time of the accelerator per year, nominal beam parameters ${ }^{6}$ at $500 \mathrm{GeV}$ and a DID-like magnetic field configuration. The not instrumented areas are for the outgoing beam (left) and the incoming beam (right).

\section{Sensor Materials under Investigation}

The FCAL Collaboration investigates several materials as possible sensors for the BeamCal. Requirements are: 
- radiation hardness against a total ionizing dose of up to $10 \mathrm{MGy}$

- a linearity of the response over at least 4 orders of magnitude of particle fluences

- if possible, no additional cooling or other support to keep the required compactness of BeamCal.

\subsection{CVD Diamond}

Chemical Vapor Deposited diamond can be produced as a polycrystalline material on wafer sizes. It is under investigation since several years ${ }^{7}$ and is very radiation hard. We investigate pCVD diamond material from the manufacturers Element 6 and the Fraunhofer Institute for Applied Solid State Physics IAF. The samples are of about $1 \mathrm{~cm}^{2}$ size and typically $300-$ $500 \mu \mathrm{m}$ thick. The properties of good classified samples are a high resistivity of about $10^{1} 5 \Omega \mathrm{cm}$ and a detectable signals of minimum ionizing particles using a part of the spectrum of a $\mathrm{Sr}^{9} 0$ source. The signal is usually quantified as the Charge Collection Distance, CCD, which gives the mean drift distance of radiation induced free charge carriers. The pCVD material is not $100 \%$ efficient showing CCDs between a few ten $\mu \mathrm{m}$ of CCD up to $150 \mu \mathrm{m}$ and more. This corresponds to a measured charge of CCD $\times$ 36 eh-pairs $/ \mu \mathrm{m}$. The dependence of the CCD on the applied electric field saturates at about $1 \mathrm{~V} / \mu \mathrm{m}$. In a hadron test beam at the CERN PS it could be shown that the linearity of the response of this material is within $30 \%$ over a range of $1 \mathrm{MIP}$ to $10^{6} \mathrm{MIPs} /\left(\mathrm{cm}^{2} 10 \mathrm{~ns}\right)$.

In a high dose irradiation test beam at the S-DALINAC of the technical university of Darmstadt it was possible to show that after 7 MGy of absorbed total ionizing dose from a $10 \mathrm{MeV}$ electron beam, the sensors were still operational. The dark current of the samples increased slightly but still remains on the level of a few pA at $500 \mathrm{~V}$. The overall signal size decreased significantly during this irradiation.

First tests of a monocrystalline sensor of about $0.25 \mathrm{~cm}^{2}$ size and $340 \mu \mathrm{m}$ thickness show a $100 \%$ efficient charge collection at low electric fields of about $0.2 \mathrm{~V} / \mu \mathrm{m}$. The leakage current was below the sensitivity of our setup ( $110-13 \mathrm{~A})$. This material has very promising properties but as of today it is only available in sizes of less than $1 \mathrm{~cm}^{2}$. 


\subsection{GaAs, SiC and Radiation Hard Silicon as Sensor Material}

Gallium arsenide is a semiconducting material with a band gap of about $1.42 \mathrm{eV}$ with a higher radiation hardness than silicon. The Siberian Institute of Technology was able to supply us with sensor prototypes in a realistic BeamCal shape, featuring 88 pads of about $5 \mathrm{~mm} \times 5 \mathrm{~mm}$ size and a thickness of $500 \mu \mathrm{m}$. The segmentation of the material is done by a metallization in the desired structure, the same procedure as used for diamond sensors. An individual pad shows a typical leakage current of $1 \mu \mathrm{A}$ at $500 \mathrm{~V}$. The CCD of the not irradiated material is about $250 \mu \mathrm{m}$ at $0.4 \mathrm{~V} / \mu \mathrm{m}$. It is noteworthy that due to the high density of the material the induced signal from a MIP is about a factor of two higher than for silicon. First results from the high dose irradiation test beam show a degradation of the signal after about $1 \mathrm{MGy}$ and an increase of the leakage current by about a factor of two. A first prototype of a $\mathrm{SiC}$ sensor was not usable as a detector for MIPs, due to the low resistivity. A prototype of a radiation hard silicon sensor made of $\mathrm{mCz}$ material was measured in laboratory and in the test beam. Without using further means of cooling the signal to noise ratio started to decrease until after about $100 \mathrm{kGy}$ no signal was detectable due to the high leakage currents at room temperature. The signal size remained constant until then.

\section{Conclusion}

BeamCal is a very important part of the instrumentation in the very forward region of the ILC detectors. One of the challenges for this electromagnetic sampling calorimeter is the development of radiation hard sensors, which are able to survive the expected level of total ionizing dose of up to $10 \mathrm{MGy}$ per year. The FCAL collaboration investigates several different possible materials. Sensor prototypes made of poly- and monocrystalline CVD diamonds, radiation hard silicon, GaAs and $\mathrm{SiC}$ are under test. These tests include laboratory measurements as current-voltage characteristics, response to minimum ionizing particles and the dependence of the response on parameters like the applied electric field or the absorbed dose. Test beam measurements prove the linearity of the response of pCVD diamond up to highest particle fluences of $10^{6}$ particles per $\mathrm{cm}^{-2} \mathrm{~s} 10 \mathrm{~ns}^{-1}$. High dose irradiation show that these samples are operational after up to $7 \mathrm{MGy}$ of absorbed dose. GaAs samples show a significant decrease of their response after about $1 \mathrm{MGy}$ and an increase of their leakage current by about a 
factor of two. First tests using radiation hard silicon show that additional measures, like e.g. cooling, have to be considered to operate the sensors to doses of $100 \mathrm{kGy}$ and beyond. A SiC prototype showed no detectable MIP response. We will investigate such a material if it will be possible to obtain material with a higher intrinsic resistivity.

\section{Acknowledgments and Appendices}

The author would like to thank all involved members of the FCAL collaboration. The Fraunhofer Institute IAF and the GSI in Darmstadt. Furthermore the Siberian Institute for Technology and the BTU Cottbus. Also thanks to the S-DALINAC crew of the technical university of Darmstadt and the CERN staff for their support during our test beams. This work was partly supported by EUDET.

\section{References}

1. ILC Global Design Effort and World Wide Study, The ILC Reference Design Report, 2007, available at http://www.linearcollider.org/.

2. The FCAL Collaboration, http://www-zeuthen.desy.de/ILC/fcal/.

3. The LDC Group, The Detector Outline Document for the Large Detector Concept, 2006, available at http://www.ilcldc.org/.

4. H. Abramowicz et al., Instrumentation of the Very Forward Region of a Linear Collider Detector, IEEE transactions of Nuclear Science, 51, p, 29832004

5. B. Parker and A. Seryi, Compensation of the effects of a detector solenoid on the vertical beam orbit in a linear collider, Phys. Rev. ST Accel. Beams 8, 041001 (2005).

6. T. Raubenheimer et al., Suggested ILC Beam Parameter Ranged, available at http://www-project.slac.stanford.edu/ilc/acceldev/beamparameters.html.

7. H. Kagan, Radiation Hard Diamond Pixel Detectors, these proceedings. 